\title{
Hemastatic biomarkers as prognostic factors in locoregionally advanced nasopharyngeal carcinoma treated with nimotuzumab plus chemoradiotherapy
}

Jianda Sun

Meizhou People's Hospital

Zhijie Chen

Meizhou People's Hospital

Jun Xiong

Meizhou People's Hospital

Hanxiong Zhang

Meizhou People's Hospital

Yibiao Chen ( $\nabla$ cybhtyy812@163.com )

https://orcid.org/0000-0002-9268-4950

Zhixiong Zhong

Meizhou People's Hospital

Research article

Keywords: Nasopharyngeal carcinoma, Chemoradiotherapy, Nimotuzumab, Coagulation assay, Efficacy

Posted Date: October 4th, 2019

DOI: https://doi.org/10.21203/rs.2.15727/v1

License: () (1) This work is licensed under a Creative Commons Attribution 4.0 International License.

Read Full License 


\section{Abstract}

Background: The prognostic value of hemostatic biomarkers in many tumors are well recognized. However, it remains unclear in locoregionally advanced nasopharyngeal carcinoma (NPC) patients treated with nimotuzumab plus chemoradiotherapy . In this study, we investigated pretreatment hemostatic biomarkers as prognostic factors for short-term efficacy after treatment. Methods: In total, 101 patients with stage $\Downarrow$ or stage $\llbracket a$ NPC were enrolled from March 2016 to December 2018. All patients received chemoradiotherapy with concurrent nimotuzumab (200 mg/week) administration. Platelet count (PLT), prothrombin time (PT), international normalized ratio (INR), thrombin time (TT), activated partial thromboplastin time (APTT), and fibrinogen (FIB) were collected before treatment. Results: There was a statistically significant association between APTT and gender $(P=0.006)$, while PT and INR were found to be correlated with age ( $P=0.020$ and $P=0.011$, respectively). Prolonged PT or elevated INR was associated with a higher $C R$ rate after treatment $(P=0.008$ and $P=0.003$, respectively). Binary logistic regression analysis demonstrated that age, T category, $\mathrm{N}$ category, PT and INR were independent prognostic factors for primary tumor response, whereas $\mathrm{N}$ category and the overall stage could independently predict cervical lymph node response. Conclusion: Pretreatment PT and INR may serve as useful prognostic biomarkers for predicting the primary tumor response to combined nimotuzumab and chemoradiotherapy in patients with locoregionally advanced NPC.

\section{Background}

Nasopharyngeal carcinoma (NPC) is a unique malignancy of the head and neck, with distinct geographical and ethnical differences, which is endemic in Southeast Asia and Southern China [1]. NPC is a highly radiosensitive and chemosensitive tumor. Radiotherapy is the mainstay for early-stage disease, while concurrent chemoradiotherapy with or without neoadjuvant chemotherapy or adjuvant chemotherapy is recommended for locoregionally advanced disease. Currently, the prognosis of patients with NPC depends primarily on TNM staging system. However, patients with the same stage and similar treatment strategy may undergo various clinical courses and have different clinical outcomes, due to the heterogeneity of tumor. Hence, TNM staging system is insufficient for the prognostication for patients with NPC.

There is a strong correlation between cancer and aberrant hemostasis [2]. Cancer cells activate the coagulation system, meanwhile, the hemostatic factors play an important role in tumor progression [3]. A substantial amount of data suggests that a hypercoagulable state is associated with a large tumor burden, clinical progression, low rates of response to chemotherapy, and a poor prognosis. In this case, hemostatic biomarkers may serve as a potential tool in predicting cancer outcomes [4]. Mounting evidence shows that hemostatic biomarkers are associated with prognosis in NPC [5-7], and help to improve the prediction accuracy of the TNM staging system [8].

Huang et al. [9] reported a multi-center phase $₫$ clinical trial of nimotuzumab combined with radiotherapy for locoregionally advanced NPC. Its results demonstrated that the complete response of the combined 
therapy group was significantly higher than that of the radiotherapy alone group, indicating that nimotuzumab synergized with radiotherapy presumably by increasing the radiosensitivity of tumor cells. Thus, nimotuzumab is recommended as an adjunct to radiotherapy for locoregionally advanced NPC. Subsequently, several retrospective researches confirmed that combining nimotuzumab with chemoradiotherapy increased treatment efficacy and improved prognosis with tolerable toxicities [10-12]. However, it is important to note that not all patients benefit from the concurrent treatment of nimotuzumab. So, the challenge is to develop a reliable and preferably non-invasive methodology to screen patients for nimotuzumab. The aim of this retrospective study is to evaluate the prognostic potential of pretreatment hemostatic biomarkers for short-term efficacy in locoregionally advanced NPC patients treated with nimotuzumab, and find the best combination of prognostic markers to identify suitable patients for this additional treatmen

\section{Methods}

\section{Patients and pretreatment evaluation}

Between March 2016 and December 2018, 101 histologically approved patients with nondisseminated NPC were hospitalized and treated in Meizhou People's Hospital. All patients were staged according to the 8th edition of the American Joint Committee on Cancer (AJCC) staging system for NPC. The eligible

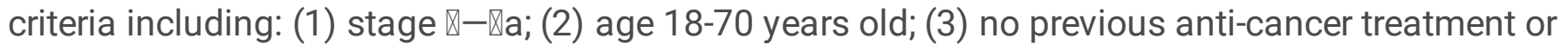
synchronous malignancy; (4) no comorbidity that is unsuitable for radiotherapy and chemotherapy; (5) complete data of coagulation assay before treatment; (6) no infection or hematology disorders; (7) no history of venous thrombosis or anticoagulation therapy during the 3 months before treatment. All patients had histologically confirmed undifferentiated non-keratinizing carcinoma, and were treated with nimotuzumab combined with chemoradiotherapy. The pretreatment staging work-up included detailed medical history, physical examination, biochemistry profiles, fiberoptic nasopharyngoscopy, computed tomography (CT) scans of the chest and upper abdomen, magnetic resonance imaging (MRI) of the nasopharynx and neck, and bone scintigram or whole body positron emission tomography/CT (PET/CT). The characteristics of the patients are listed in Table 1. Written informed consent was obtained from all patients. This study was approved by the Ethics Board of the Meizhou People's Hospital.

\section{Hemastatic biomarkers evaluation}

A $4 \mathrm{~mL}$ blood sample was collected before treatment, and then the hemastatic biomarkers, such as PLT, PT, INR, TT, APTT and FIB, were assessed automatically using an analyzer SYSMEX XE-2100 and an analyzer WERFEN ACL TOP according to the instructions provided by the manufacturers.

\section{Treatment}


Of the 101 patients, 61 (60.4\%) received radical radiotherapy with intensity modulated radiotherapy (IMRT) technique, while the rest with volumetric arc therapy (VMAT) technique. The prescribed doses were 70-74 Gy to the primary disease in 2.12-2.24 Gy per fraction with five daily fractions per week, 66-74 Gy to the positive cervical lymph nodes in 2.0-2.24 Gy per fraction, 60-66 Gy to the high risk planning target volume in 1.82-2.0 Gy per fraction, 50-54 Gy to the low risk planning target volume in 1.79-1.93 Gy per fraction. All patients received neoadjuvant chemotherapy, followed by concurrent chemoradiotherapy. Neoadjuvant chemotherapy consisted of 1-3 cycles of platinum-based combined regimens administered every 3 weeks. Concurrent chemotherapy was platinum alone for 1-3 cycles. Adjuvant chemotherapy was recommended to those with detectable plasma EBV DNA or residual disease after radiotherapy, T4 and/or N3 disease (23 patients). 16 patients received adjuvant chemotherapy consisted of 1-3 cycles of platinum-based combined regimens. Those who were unable to tolerate the intravenous chemotherapy, would received oral capecitabine or S-1 (7 patients). Nimotuzumab was administered concomitantly with radiotherapy at a dose of $200 \mathrm{mg}$ weekly. Among them, 15 patients (14.9\%) received 6 cycles of nimotuzumab, while the rest received 7 cycles.

\section{Treatment responses assessment}

At the end of radiotherapy, fiberoptic nasopharyngoscopy, chest X-ray/CT, ultrasound/CT of upper abdomen, MRI of the nasopharynx and neck were performed. Treatment responses were assessed according to the Response Evaluation Criteria in Solid Tumors (version 1.1) [13].

\section{Statistical analysis}

Continuous variables were categorized using median values as cut-off point. Assessments of association of pretreatment hemastatic biomarkers with clinicopathological features, and short-term efficacy after treatment, were accomplished using Chi-square test. Binary logistic regression analysis was carried out to determined the significant prognostic factors for short-term efficacy. All statistical analysis was performed using SPSS 25.0 software, and $P$ values of $<0.05$ were considered statistically significant.

\section{Results}

\section{The correlations between pretreatment hemastatic biomarkers and clinicopathological features}

There was a statistically significant association between APTT and gender. Male patients exhibited prolonged APTT when compared with female subjects $(P=0.006$; Table 2$)$. Patients aged $\leq 47$ had significant prolonged PT and elevated INR than those aged $>47(P=0.020$ and $P=0.011$, respectively). Advanced $\mathrm{N}$ category was associated with elevated platelet counts and INR, although the differences between two groups nearly reached statistical significance $(P=0.066$ and $P=0.064$, respectively). However, neither pretreatment TT nor FIB was correlated with any clinicopathological features of patients. 


\section{The correlations between pretreatment hemastatic biomarkers and short-term efficacy}

At the end of radiotherapy, the response rates of the primary disease were as follows: 69 patients had a complete response (CR) (68.3\%), 30 patients had a partial response (PR) $(29.7 \%)$, and 2 patients had a stable disease (SD) (2.0\%), giving an objective response rate of $98.0 \%$. For 97 NPC patients with lymphoadenopathy, CR, PR, and SD rates of the cervical lymph nodes were $80.4 \%$ (78/97), 18.6\% (18/97), $1.0 \%$ (1/97), respectively. As only 3 patients had a stable disease after radiotherapy, they were combined with those who had a partial response, when the association between pretreatment hemastatic biomarkers and tumor response was evaluated. At the end of study, $81.3 \%$ of patients with PT $>11.2 \mathrm{sec}$ and $56.6 \%$ of patients with PT $\leq 11.2$ sec experienced CR of primary tumor after treatment $(P=0.008$; Table 3). The CR rate of patients with INR $₫ 1.05$ was significantly higher than that of patients with INR $\leq 1.05$ (83.0\% vs. $55.6 \%, P=0.003)$. No significant correlations were observed between the short-term efficacy and pretreatment PLT, TT, APTT, or FIB.

\section{Prognostic factors}

Binary logistic regression analysis demonstrated that age, T category, N category, PT and INR were independent prognostic factors for primary tumor response. Younger age, early $\mathrm{T}$ category, advanced $\mathrm{N}$ category, prolonged PT and elevated INR were associated with a higher response rate of primary tumor. N category and overall stage were independent prognostic factors for cervical lymph nodes response. Patients with advanced $\mathrm{N}$ category or overall stage would have relatively lower response rates of cervical lymph nodes (Table 4).

\section{Discussion}

Malignancy affects the hemastatic system, and in turn, the hemastatic system affects malignancy [3]. Subclinical hypercoagulation condition is common in cancer patients, and almost all patients with advanced tumors present with low-grade systemic coagulation activation, as indicated by abnormalities in laboratory coagulation tests [4, 14]. Meanwhile, systemic activation of hemostasis has been exhaustively implicated in the pathogenesis, progression, and metastasis of cancer [15]. In the current analysis, advanced $\mathrm{N}$ category was associated with aberrant hemostasis (i.e. elevated platelet counts and INR), and the differences were nearly reached statistical significance.

PT is the time taken for plasma to clot when exposed to tissue factors. It is often used to evaluate the extrinsic pathway of coagulation, and reflects the activity of coagulation factors $\nabla, \nabla, \nabla, \nabla$, and $\nabla$. Given the results are coming from different instruments and reagents, it is difficult to compare the PT measurements among different laboratories. Nonetheless, INR makes the results comparable. Mounting 
evidence demonstrated that prolonged PT was associated with poor prognosis in lung cancer [16,17], pancreatic cancer [18], colorectal cancer [19], and hepatocellular carcinoma [20]. The exact mechanisms underlying this association remain unclear. One of the possible explanations is that the activation of the blood clotting cascade is common in the tumor microenvironment [21], and systemic activation of hemostasis and thrombosis promotes the progression and metastasis of cancer [15]. During this process, a large number of coagulation factors may be consumed, resulting in the prolongation of PT. In the current study, our result showed that pretreatment PT and INR were independent prognostic factors for primary tumor response. There was a significantly positive correlation between PT/INR and the response extent of primary tumor. Interestingly, those with prolonged PT or elevated INR had a higher CR rate after treatment. We speculate on the reasons for the discrepancy between others' findings and ours. Firstly, NPC has distinct biological characteristics from other solid tumors, so that the prognostic factors may be different; Secondly, chemoradiotherapy is the standard treatment modality for locoregionally advanced NPC currently, whereas patients in this retrospective study received combined nimotuzumab with chemoradiotherapy, so that the treatment outcomes may be changed with the addition of nimotuzumab. Several previous studies concluded that nimotuzumab combined with chemoradiotherapy benefited patients, and was more effective than chemoradiotherapy alone[10-12]. According to our findings, prolonged PT and elevated INR before treatment can predict short-term efficacy in locoregionally advanced NPC patients for combing nimotuzumab treatment with chemoradiotherapy.

It is well known that platelets play a vital role in hemostasis and thrombosis. But mounting evidence suggests that platelets also mediate tumor cell growth, dissemination, and angiogenesis. Platelets facilitate tumor metastases through direct interaction with tumor cells or through secreting bioactive proteins. Platelets also form aggregates with tumor cells, thereby protecting them from host immune surveillance. Meanwhile, platelet-secreted growth factors stimulate tumor cells proliferation to micrometastatic foci [22-23]. FIB is a key glycoprotein synthesized by hepatocytes, which can turn into fibrin by reacting with thrombin. Fibrin provides a scaffold for tumor cell anchorage and invasion, and protects tumor cells from immune system recognition and destruction [4]. Several studies have confirmed that an increase in platelet number and FIB level were correlated with poor prognosis in many solid tumor types [24-29]. A similar conclusion was also drawn in NPC [5-6, 30-33]. Thrombocytosis and hyperfibrinogenemia were found to be unfavorable prognostic factors. In addition, Gao et al. [30] reported that platelet count showed significant correlation with $\mathrm{T}$ stage and clinical stage, while $\mathrm{He}$ et al. [6] showed that a high pretreatment plasma FIB level was associated with advanced TNM stage. Nevertheless, in the current study, neither pretreatment PLT nor FIB level was significantly associated with TNM stage, or tumor response after radiotherapy. One of the possible explanations is that the assay process is not harmonized across different laboratories, and cut-off values for variables are determined by different methods, leading to different results. In most of the published studies, cut-off values were established using receiver operating characteristic (ROC) curve analysis or upper limit of normal. However, continuous variables were categorized using median values as a cut-off point in the present analysis. Due to the lack of assay standardization, the cut-off points have varied across reported studies, 
making it difficult to compare the results across studies [34]. Hence, seeking a harmonized assay with standard procedures is warranted.

Several limitations of the present study should be noted. Firstly, the study was a retrospective cohort study conducted at a single center with a relatively small sample size. Secondly, plasma D-dimer, an important hemostatic biomarker, was not included in the present study. Previous study has shown that elevated pretreatment plasma D-dimer level was a highly significant predictor for a worse outcome in patients with NPC [7]. Thirdly, only short-term tumor response after radiotherapy was evaluated in our study, but long-term survival outcomes are yet to be followed up. Fourthly, as some patients declined MRI three months after radiotherapy, the results were only evaluated at the end of treatment with no follow-up evaluation. In this case, the prognostic values of hemostatic biomarkers might have been underestimated.

\section{Conclusions}

In summary, our results suggest that pretreatment PT and INR were independent prognostic factors for the primary tumor response. There was a significant positive correlation between PT/INR and the response extent of primary tumor. PT and INR are routine blood-based parameters that are extremely reproducible and do not cause additional cost or laborious efforts, which makes them ideal biomarkers for predicting the response to combined nimotuzumab and chemoradiotherapy in patients with locoregionally advanced NPC.

\section{Abbreviations}

APTT: Activated partial thromboplastin time; CT: Computed tomography; CR: Complete resoponse; FIB: Fibrinogen; INR: International normalized ratio; IMRT: Intensity modulated radiotherapy; MRI: Magnetic resonance imaging; NPC: Nasopharyngeal carcinoma; PLT: Platelet count; PT: Prothrombin time; PET/CT: positron emission tomography/CT; PR: Partial response; ROC: Receiver operating characteristic; SD: Stable disease; TT: Thrombin time; VMAT: volumetric arc therapy

\section{Declarations}

\section{Acknowledgments}

We are grateful to the patients who participated in this study.

\section{Funding}

This study was supported by the Medical and Health Research Program of Meizhou City, China [grant number 2017-B-20]. The funding agency had no role in the study design, data collection, analysis, 
interpretation, or preparation of the manuscript.

\section{Availability of data and materials}

The datasets used in the current study are available from the corresponding author on reasonable request.

\section{Authors' contributions}

JDS conceived and designed the study, carried out data collection, data analysis, interpretation of the results, and the drafting of the manuscript. ZJC participated in data collection. JX and HXZ reviewed MR images. YBC and ZXZ coordinated the study, and revised the manuscript. All authors read and approved the final manuscript.

\section{Ethics approval and consent to participate}

This study was approved by the Ethics Board of the Meizhou People's Hospital. Written informed consent was obtained from all patients.

\section{Consent for publication}

Not applicable.

\section{Competing interests}

The authors declare that they have no competing interests.

\section{Publisher's Note}

Springer Nature remains neutral with regard to jurisdictional claims in published maps and institutional affiliations. 
${ }^{1}$ Department of Radiation Oncology, Meizhou People's Hospital, Meizhou 514031, China. ${ }^{2}$ Cadiovascular center, Meizhou People's Hospital, Meizhou 514031, China

\section{References}

[1] Wee JT, Ha TC, Loong SL, Qian CN. Is nasopharyngeal cancer really a "Cantonese cancer"? Chin J Cancer. 2010; 29(5): 517-26.

[2] van den Berg YW, Osanto S, Reitsma PH, Versteeg HH. The relationship between tissue factor and cancer progression: insights from bench and bedside. Blood. 2012; 119(4): 924-32.

[3] Falanga A, Marchetti M, Vignoli A. Coagulation and cancer: biological and clinical aspects. J Thromb Haemost. 2013; 11(2): 223-33.

[4] Falanga A, Marchetti M. Hemostatic biomarkers in cancer progression. Thromb Res. 2018; 164 Suppl 1: S54-S61.

[5] Chen YP, Chen C, Mai ZY, Gao J, Shen LJ, Zhao BC, et a1. Pretreatment platelet count as a predictor for survival and distant metastasis in nasopharyngeal carcinoma patients. Oncol Lett. 2015; 9(3): 14581466.

[6] He SS, Wang Y, Yang L, Chen HY, Liang SB, Lu LX, et a1. Plasma Fibrinogen Correlates with Metastasis and is Associated with Prognosis in Human Nasopharyngeal Carcinoma. J Cancer. 2017; 8(3): 403-409.

[7] Chen WH, Tang LQ, Wang FW, Li CP, Tian XP, Huang XX, et a1. Elevated levels of plasma D-dimer predict a worse outcome in patients with nasopharyngeal carcinoma. BMC Cancer. 2014; 14: 583.

[8] Chen YP, Zhao BC, Chen C, Shen LJ, Gao J, Mai ZY, et a1. Pretreatment platelet count improves the prognostic performance of the TNM staging system and aids in planning therapeutic regimens for nasopharyngeal carcinoma: a single-institutional study of 2,626 patients. Chin J Cancer. 2015; 34(3): 137-46.

[9] Huang XD, Yi JL, Gao L, Xu GZ, Jin J, Yang WZ, et a1. Multi-center phase II clinical trial of humanized anti-epidermal factor receptor monoclonal antibody h-R3 combined with radiotherapy for locoregionally advanced nasopharyngeal carcinoma. Zhonghua Zhong Liu Za Zhi. 2007; 29(3): 197-201.

[10] You R, Hua YJ, Liu YP, Yang Q, Zhang YN, Li JB, et a1. Concurrent Chemoradiotherapy with or without Anti-EGFR-Targeted Treatment for Stage II-IVb Nasopharyngeal Carcinoma: Retrospective Analysis with a Large Cohort and Long Follow-up. Theranostics. 2017; 7(8): 2314-2324.

[11] Wang F, Sun Q, Jiang C, Liu T, Rihito A, Masoto S, et a1. Additional induction chemotherapy to concurrent chemotherapy and intensity-modulated radiotherapy with or without nimotuzumab in first-line 
treatment for locoregionally advanced nasopharyngeal carcinoma: a propensity score matched analysis. J Cancer. 2018; 9(3): 594-603.

[12] Li Z, Li Y, Yan S, Fu J, Zhou Q, Huang X, et a1. Nimotuzumab combined with concurrent chemoradiotherapy benefits patients with advanced nasopharyngeal carcinoma. Onco Targets Ther. 2017; 10: 5445-5458.

[13] Eisenhauer EA, Therasse P, Bogaerts J, Schwartz LH, Sargent D, Ford R, et a1. New response evaluation criteria in solid tumours: revised RECIST guideline (version 1.1). Eur J Cancer. 2009; 45(2): 228-47.

[14] Langer F, Bokemeyer C. Crosstalk between cancer and haemostasis. Implications for cancer biology and cancer-associated thrombosis with focus on tissue factor. Hamostaseologie. 2012; 32(2): 95-104.

[15] Repetto O, De Re V. Coagulation and fibrinolysis in gastric cancer. Ann N Y Acad Sci. 2017; 1404(1): 27-48.

[16] Tas F, Kilic L, Serilmez M, Keskin S, Sen F, Duranyildiz D. Clinical and prognostic significance of coagulation assays in lung cancer. Respir Med. 2013; 107(3): 451-7.

[17] Zhu M, Dai Y, Gao F, Xu C, Chen L, Xu Y, et a1. Correlations of coagulation indexes and inflammatory changes with the prognosis of lung cancer complicated with thromboembolic disease. J BUON. 2019; 24(2): 585-590.

[18] Zhang K, Gao HF, Mo M, Wu CJ, Hua YQ, Chen Z, et a1. A novel scoring system based on hemostatic parameters predicts the prognosis of patients with advanced pancreatic cancer. Pancreatology. 2019; 19(2): 346-351.

[19] Lee S, Huh SJ, Oh SY, Koh MS, Kim SH, Lee JH, et a1. Clinical significance of coagulation factors in operable colorectal cancer. Oncol Lett. 2017; 13(6): 4669-4674.

[20] Wang XP, Mao MJ, He ZL, Zhang L, Chi PD, Su JR, et a1. A retrospective discussion of the prognostic value of combining prothrombin time(PT) and fibrinogen(Fbg) in patients with Hepatocellular carcinoma. J Cancer. 2017; 8(11): 2079-2087.

[21] Lima LG, Monteiro RQ. Activation of blood coagulation in cancer: implications for tumour progression. Biosci Rep. 2013; 33(5).

[22] Sharma D, Brummel-Ziedins KE, Bouchard BA, Holmes CE. Platelets in tumor progression: a host factor that offers multiple potential targets in the treatment of cancer. J Cell Physiol. 2014; 229(8): 100515.

[23] Schlesinger M. Role of platelets and platelet receptors in cancer metastasis. J Hematol Oncol. 2018; 11(1): 125. 
[24] Holgersson G, Bergstrom S, Hallqvist A, Liv P, Nilsson J, Willen L, et a1. The prognostic value of pretreatment thrombocytosis in two cohorts of patients with non-small cell lung cancer treated with curatively intended chemoradiotherapy. Neoplasma. 2017; 64(6): 909-915.

[25] Wang YH, Kang JK, Zhi YF, Zhang Y, Wang ZQ, Zhou Q, et a1. The pretreatment thrombocytosis as one of prognostic factors for gastric cancer: A systematic review and meta-analysis. Int J Surg., 2018; 53: 304-311.

[26] Wang H, Gao J, Bai M, Liu R, Li H, Deng T, et a1. The pretreatment platelet and plasma fibrinogen level correlate with tumor progression and metastasis in patients with pancreatic cancer. Platelets. 2014; 25(5): 382-7.

[27] Lee S, Huh SJ, Oh SY, Koh MS, Kim SH, Lee JH, et a1. Clinical significance of coagulation factors in operable colorectal cancer. Oncol Lett. 2017; 13(6): 4669-4674.

[28] Wen J, Yang Y, Ye F, Huang X, Li S, Wang Q, et a1. The preoperative plasma fibrinogen level is an independent prognostic factor for overall survival of breast cancer patients who underwent surgical treatment. Breast. 2015; 24(6): 745-50.

[29] Zhao K, Deng H, Qin Y, Liao W, Liang W. Prognostic significance of pretreatment plasma fibrinogen and platelet levels in patients with early-stage cervical cancer. Gynecol Obstet Invest. 2015; 79(1): 25-33.

[30] Gao J, Zhang HY, Xia YF. Increased platelet count is an indicator of metastasis in patients with nasopharyngeal carcinoma. Tumour Biol. 2013; 34(1): 39-45.

[31] Xie X, Zeng X, Cao S, Hu X, Shi Q, Li D, et a1. Elevated pretreatment platelet distribution width and platelet count predict poor prognosis in nasopharyngeal carcinoma. Oncotarget. 2017; 8(62): 106089106097.

[32] Lan M, Chen C, Huang Y, Mao M, Han F, Liao J, et a1. Elevated plasma fibrinogen level shows superior prognostic value than Epstein-Barr virus DNA load for stage IVA/B nasopharyngeal carcinoma patients in the intensity-modulated radiotherapy era. Oncotarget. 2016; 7(29): 46242-46252.

[33] Tang LQ, Chen QY, Guo SS, Chen WH, Li CF, Zhang L, et a1. The impact of plasma Epstein-Barr virus DNA and fibrinogen on nasopharyngeal carcinoma prognosis: an observational study. $\mathrm{Br} \mathrm{J}$ Cancer. 2014; 111(6): 1102-11.

[34] Kim KY, Le QT, Yom SS, Ng RHW, Chan KCA, Bratman SV, et a1. Clinical Utility of Epstein-Barr Virus DNA Testing in the Treatment of Nasopharyngeal Carcinoma Patients. Int J Radiat Oncol Biol Phys. 2017; 98(5): 996-1001.

\section{Tables}

Due to technical limitations, the tables have been placed in the Supplementary Files section. 


\section{Supplementary Files}

This is a list of supplementary files associated with this preprint. Click to download.

- Table1.docx

- Table3.docx

- Table2.docx

- Table4.docx 